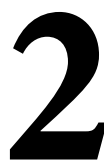

\title{
EL ACCESO A LA PROFESIÓN DOCENTE EN ESPAÑA EN PERSPECTIVA EUROPEA. ALGUNAS REFLEXIONES ORIENTADAS A LA MEJORA DE LA SELECCIÓN DEL PROFESORADO
}

\author{
(ACCESS TO TEACHING PROFESSION IN SPAIN IN AN EUROPEAN \\ PERSPECTIVE. SOME REFLECTIONS TO IMPROVE THE SELECTION \\ OF TEACHERS)
}

Inmaculada Egido Gálvez

Universidad Complutense de Madrid

\section{RESUMEN}

Desde hace tiempo, una de las cuestiones mejor documentadas por la investigación educativa es que el profesorado constituye un factor clave para la calidad de la enseñanza. Por esa razón, la búsqueda de procedimientos adecuados para la selección de los futuros docentes es una materia de vital importancia en todos los sistemas educativos, aunque en España se trata de una temática escasamente trabajada. El objetivo de este artículo es contribuir a un mejor conocimiento del funcionamiento de los sistemas de selección y acceso a la docencia vigentes en nuestro país. Para ello, en primer lugar, se presenta una panorámica de los procedimientos de selección empleados en Europa, con el fin de contextualizar el caso español. Tras ese marco contextual, se analiza el sistema español, tomando como referencia un conjunto de trabajos en los que se reflejan las opiniones que expresan sobre este tema tanto los propios profesores como una serie de expertos de ámbito nacional e internacional. A partir de esas informaciones, el artículo propone algunas líneas de reflexión que deberían ser consideradas para mejorar en el futuro la selección del profesorado en nuestro país.

\section{ABSTRACT}

For a long time, one of the best documented issues of the educational research is that teachers are a key factor for the quality of education. Thus, the search of effective teachers selection procedures is a very important subject in all education systems, although in Spain this shows to be a scarcely developed is- 
sue. The objective of this article is to contribute to a better knowledge of the running of the teacher selection and access systems in our country. At the first stage the analysis shows a perspective of the current procedures of selection used in Europe, in order to compare them with the Spanish case. After this contextual framework, Spanish system is analysed, taking into consideration some studies that deal with opinions of Spanish teachers and national and international experts in education. As a consequence of this information, the article proposes some reflection lines that should be considered to improve the selection of teachers in our country.

\section{LA SELECCIÓN DEL PROFESORADO Y LA CALIDAD DE LA ENSEÑNANA}

"La calidad de un sistema educativo no puede ser mejor que la de su profesorado" (Barber; Mourshed, 2007). Esta frase, tomada del conocido informe McKinsey, que analiza las claves del éxito de los países líderes en el proyecto PISA, ha puesto de manifiesto, una vez más, el papel clave de los profesores en la educación. De las tres variables que tienen en común sistemas tan dispares como Finlandia, Hong Kong, Corea del Sur y Canadá, dos hacen referencia precisamente al profesorado: son los que consiguen que los mejores alumnos estudien para ser profesores y los que proporcionan a esos profesores las mayores oportunidades de desarrollo profesional. En realidad, el resultado de este informe no supone una sorpresa, sino que viene a confirmar una evidencia que la investigación educativa ha confirmado reiteradamente: los profesores son un factor fundamental en el aprendizaje, no sólo desde el punto de vista del rendimiento académico de los estudiantes, sino también en lo que se refiere a la motivación de éstos hacia el aprendizaje futuro (Darling-Hammond, 2000).

No es de extrañar, por tanto, que en estos momentos, en los que la preocupación por la calidad de la educación es una constante en todos los sistemas educativos europeos, buena parte de los esfuerzos de las autoridades responsables de los mismos se dediquen a hacer realidad el desafío que tanto la Organización para la Cooperación y el Desarrollo Económico (OCDE) como la propia Unión Europea (UE) han resumido en tres palabras: atraer, formar y retener al profesorado (Eurydice, 2004; ODCE, 2004). Es necesario conseguir un número suficiente de buenos candidatos para la docencia, proporcionarles una formación adecuada y procurar que tengan unas condiciones laborales lo suficientemente atractivas como para mantener la motivación durante toda su vida profesional. En los países miembros de la UE, este triple reto se ha traducido, en los años pasados, en la puesta en práctica de un conjunto de reformas que han modificado tanto los sistemas de formación inicial y continua de los docentes como sus condiciones laborales, configurando los nuevos modelos de profesión docente en Europa (Eurydice, 2005). 
Un aspecto sustancial dentro de esos modelos lo constituyen los sistemas de selección y acceso a la profesión docente. Aunque mucho menos analizados que otras condiciones de trabajo, como los horarios o las retribuciones salariales, los mecanismos empleados para tratar de garantizar que quienes acceden a la docencia puedan cumplir adecuadamente con su tarea condicionan también el perfil profesional del profesorado en los distintos países. Los sistemas de selección actúan como "filtro" para el acceso a la enseñanza y, por tanto, indican, aunque sea indirectamente, qué modelo de profesor se considera adecuado en cada país.

En la gran mayoría de los sistemas de nuestro entorno, lo habitual es que los procesos selectivos para la docencia se basen en la preparación académica de los aspirantes, primando, por tanto, el dominio cognoscitivo. Sin embargo, hace ya tiempo que los expertos reclaman la necesidad de garantizar otras condiciones adicionales, relacionadas con las actitudes y las habilidades interpersonales de los futuros docentes. Por ejemplo, el informe Wall (1959) de la UNESCO señalaba ya la importancia de contar con algún tipo de pruebas referidas a la personalidad de los aspirantes a profesor, con el fin de evitar el acceso a la profesión de personas con desequilibrios o problemas emocionales que pudieran conducir al fracaso de la relación educativa o producir efectos negativos sobre los estudiantes. Desde entonces, han sido muchos los autores que se han pronunciado en esta línea (Peretti, 1982; Esteve, 1984), afirmando que un requisito imprescindible para quienes trabajan en educación debe ser la motivación y la implicación en la mejora de los aprendizajes de los alumnos, para lo que no basta únicamente con adquirir unos conocimientos determinados. Con este fin, algunos países emplean también sistemas encaminados a analizar la personalidad de los candidatos a los puestos docentes, sus actitudes hacia la enseñanza o su disponibilidad para el trabajo en determinados tipos de escuela.

En el caso de España, a pesar de la importancia que el sistema de acceso a la docencia puede tener para la calidad del sistema escolar, lo cierto es que se trata de un tema al que se ha dedicado una escasa atención. Si se exceptúan algunos trabajos de carácter histórico (Dávila, 1988; Fernández Tilve y Malvar, 2000; Gabriel, 1994; Requena, 2007), son pocos los estudios que abordan esta cuestión. No obstante, en el momento actual, en el que estamos inmersos en un proceso de reforma de la formación inicial del profesorado que, inevitablemente, tendrá repercusiones en el sistema de acceso a la profesión, conviene detenerse a reflexionar sobre esta temática.

Partiendo de esa premisa, en las páginas siguientes se intenta profundizar en los sistemas de ingreso a la docencia en España. En primer lugar, con el fin de contextualizar el caso español, se presenta una breve descripción de los modelos de selección del profesorado vigentes en los países 
miembros de la Unión Europea. Tras ese marco comparativo, se tratan los mecanismos de acceso a la profesión docente en el sistema educativo español, primando en este caso una aproximación valorativa más que descriptiva. En concreto, se presentan los resultados de dos investigaciones realizadas recientemente sobre este tema, en las que profesores y especialistas en educación han mostrado sus opiniones sobre la adecuación de los sistemas de acceso a la docencia. De manera complementaria, se sintetizan también las recomendaciones que la OCDE ha planteado a España en relación con la selección del profesorado. Partiendo de este conjunto de informaciones, en el apartado final se formulan algunas líneas de reflexión que tienen como objeto contribuir a la mejora de los procesos de selección del profesorado en nuestro país.

\section{LOS MODELOS EUROPEOS DE SELECCIÓN DEL PROFESORADO}

Cuando se analizan los procedimientos de acceso a la profesión docente en la Unión Europea, es necesario tener en cuenta que algunos países realizan una selección previa al comienzo de la formación docente. En esos países, los estudiantes que desean cursar estudios de educación deben superar un primer proceso selectivo, mediante pruebas o entrevistas diseñadas específicamente para los futuros profesores, además de cumplir con los requisitos de carácter general que se exijan para el ingreso en la enseñanza superior en cada caso. Así, Dinamarca, Eslovaquia, Estonia, Francia, Finlandia, Lituania, Luxemburgo, Polonia, Reino Unido y Rumanía llevan a cabo un proceso selectivo anterior a la formación, que restringe la entrada de los aspirantes a las Facultades o Centros de formación del profesorado. Estos sistemas limitan las plazas ofertadas por las instituciones de formación inicial y preparan a un número reducido de futuros profesores, en lugar de formar a un gran número de estudiantes y seleccionar después a algunos de ellos para acceder a un puesto de trabajo. Se trata, por tanto, de países en los que la carrera docente se inicia en el mismo momento de la entrada en la formación inicial, pudiendo existir otros procesos selectivos posteriores (Montero, 2006).

En lo que se refiere a la selección realizada con posterioridad a la formación inicial, los estudios comparados coinciden en señalar la existencia de tres grandes modelos para el acceso a la docencia en el sector público en Europa. Así, el informe realizado por la red Eurydice de la Unión Europea (2002) identifica tres procedimientos de acceso: las oposiciones, las listas de candidatos y los sistemas de contratación abierta. En una línea muy similar, el estudio llevado a cabo por la UNESCO (Murillo, 2007) presenta también tres mecanismos principales de ingreso en la docencia: la oposición, el concurso y la selección libre (tabla 1). 


\begin{tabular}{|c|c|c|}
\hline Oposición & $\begin{array}{l}\text { Lista de candida- } \\
\text { tos/concurso de méritos }\end{array}$ & Selección abierta \\
\hline $\begin{array}{c}\text { España } \\
\text { Francia }^{1} \\
\text { Grecia } \\
\text { Italia } \\
\text { Luxemburgo }^{1} \\
\text { Malta } \\
\text { Portugal } \\
\text { Rumanía }\end{array}$ & $\begin{array}{c}\text { Alemania } \\
\text { Austria } \\
\text { Bélgica (Comunidades } \\
\text { francesa y alemana) } \\
\text { Chipre }\end{array}$ & $\begin{array}{c}\text { Bélgica (Comunidades } \\
\text { flamenca y francesa) } \\
\text { Bulgaria } \\
\text { Dinamarca } \\
\text { Eslovaquia } \\
\text { Eslovenia } \\
\text { Estonia } \\
\text { Finlandia } \\
\text { Hungría } \\
\text { Irlanda } \\
\text { Islandia } \\
\text { Letonia } \\
\text { Liechtenstein } \\
\text { Lituania } \\
\text { Noruega } \\
\text { Países Bajos } \\
\text { Polonia } \\
\text { Reino Unido } \\
\text { República Checa } \\
\text { Suecia }\end{array}$ \\
\hline
\end{tabular}

${ }^{1}$ En Francia y Luxemburgo la selección se realiza antes de finalizar la formación, cuando los profesores comienzan la fase de preparación en el lugar de trabajo.

${ }_{2}$ En la Comunidad francesa de Bélgica se utiliza la lista de candidatos en los centros de la Comunidad, mientras en los centros de las provincias y los municipios se lleva a cabo un proceso de selección abierto.

Tabla 1. Sistemas de acceso a la profesión docente en la enseñanza públia en los países de la Unión Europea. (Eurydice, 2002)

Como puede observarse en la tabla, el trabajo publicado por Eurydice muestra que, en términos numéricos, son minoría los países de la Unión Europea que utilizan la oposición o las listas de candidatos para el ingreso en la docencia, mientras la mayoría tiene un sistema de contratación abierta o libre. Geográficamente, casi todos los países que utilizan la oposición pertenecen a la Europa mediterránea, mientras las listas de candidatos se emplean en algunos países de la Europa central y los procesos abiertos son lo habitual en el resto de la UE.

En la mayoría de los casos, el sistema de acceso por oposición consiste en un concurso público, organizado a nivel central, que se lleva a cabo tras la finalización de la formación inicial del profesorado. No obstante, algunos países realizan la selección antes de que los futuros profesores terminen los estudios, generalmente cuando los estudiantes inician la fase final de su preparación en los centros escolares. En todos los países que emplean 
este sistema, los resultados de los exámenes de las oposiciones se complementan con la valoración de otros méritos de los candidatos a la hora de adjudicar los puestos de trabajo.

En los países que utilizan el sistema de concurso o lista de candidatos, los aspirantes presentan su solicitud ante las autoridades correspondientes, que ordenan las candidaturas en función de los méritos acreditados y realizan la selección del número necesario para cubrir las plazas vacantes. Uno de los criterios para la elaboración de las listas suele ser la fecha de la presentación de las candidaturas, pero también se tienen en cuenta otros factores, como la experiencia docente previa, las calificaciones académicas o la formación complementaria acreditada por los aspirantes.

Por último, en los países que llevan a cabo la contratación del profesorado mediante procesos de selección de carácter abierto, los centros escolares son los responsables de elegir a sus profesores, contando, en ocasiones, con la participación de las autoridades locales para esta tarea. En estos países no existen criterios comunes para la selección del personal docente y los sistemas que se utilizan pueden estar más o menos regulados por la legislación. Los puestos disponibles se anuncian públicamente y los profesores se presentan para cubrir las vacantes que existen en un centro concreto. El procedimiento más habitual para la selección suele ser la realización de entrevistas, siendo muy frecuente que el director tenga un peso determinante en la decisión adoptada.

Estos tres grandes modelos de selección utilizados en Europa guardan relación con el grado de centralización de los distintos sistemas educativos. Así, los países de tradición más centralista optan por los dos primeros sistemas, la oposición o la lista de candidatos, mientras los más descentralizados utilizan el sistema de acceso libre. En estos países, los centros escolares disponen de un amplio margen de autonomía para contratar a los profesores que consideren más adecuados, en función de sus características, sus necesidades y su proyecto educativo.

Por lo que se refiere a la enseñanza privada, en todo el ámbito europeo está generalizado el sistema de libre contratación del profesorado, que se emplea también en los centros privados financiados por los poderes públicos. Según el país concreto que se analice, el proceso de selección recae en el director del centro escolar, en la entidad titular o en el consejo de administración de la escuela privada. La única excepción a esta tendencia es la de Francia, donde los profesores de las escuelas privadas que reciben financiación estatal son reclutados por medio de un examen, siguiendo un procedimiento equivalente al que existe para la enseñanza pública (Eurydice, 2008). Este examen, que comenzó a funcionar en 1995, otorga una certificación de- 
nominada CAFEP (Certificado de aptitud para la enseñanza en instituciones de educación privada), que habilita para ejercer la docencia en los centros privados subvencionados por el Estado.

\section{EL SISTEMA ESPAÑOL DE SELECCIÓN DEL PROFESORADO}

Atendiendo a la clasificación presentada en el apartado anterior, España se incluye en el grupo de países europeos en los que el modelo de acceso a la profesión docente en la enseñanza pública se basa en la oposición, sin que exista un sistema selectivo previo al inicio de la formación inicial. De acuerdo con la normativa actual (Real Decreto 276/2007), en el caso español el proceso de selección se organiza en dos fases:

$1^{\mathrm{a}}$. Fase de oposición. Se trata de una única prueba estructurada en dos ejercicios:

- Primer ejercicio. Tiene por objeto la demostración de los conocimientos específicos necesarios para impartir docencia.

- Segundo ejercicio. Evalúa la aptitud pedagógica y el dominio de las técnicas docentes mediante la presentación de una programación y la exposición oral de una unidad didáctica.

$2^{\mathrm{a}}$. Fase de concurso. En ella se valora la formación académica de los aspirantes y, de forma preferente, la experiencia docente previa en centros públicos de la misma etapa educativa.

Una vez seleccionados, los futuros docentes deben realizar un periodo de prácticas, que también forma parte del proceso selectivo. Superado dicho periodo, los profesores adquieren la condición de funcionarios públicos de carrera, lo que les garantiza un puesto de trabajo de carácter vitalicio.

No obstante, además del ingreso por medio del concurso-oposición, los profesores españoles también pueden acceder a la docencia en la enseñanza pública a través de los contratos de interinidad (profesores interinos). Este sistema, pensado inicialmente para cubrir las plazas vacantes o realizar las suplencias de las bajas de carácter temporal de los funcionarios, ha constituido en la práctica una vía de acceso importante en términos numéricos, puesto que ha llegado a suponer casi el $20 \%$ del total del profesorado de los centros públicos en los últimos años ${ }^{1}$.

En el sector de la enseñanza privada, la selección del profesorado recae en el titular del centro. No obstante, en los centros concertados el proceso de 
selección debe ajustarse a los principios establecidos por la Ley Orgánica 8/1985 Reguladora del Derecho a la Educación. Dicha ley dispone que las vacantes de puestos docentes que se produzcan en esos centros deben ser anunciadas públicamente y que los criterios de selección deben establecerse por acuerdo entre el consejo escolar y el titular del centro, basándose en los principios de mérito y capacidad. El consejo escolar designa una comisión de selección, integrada por el director, dos profesores y dos padres de alumnos.

Si se exceptúa el alto porcentaje de interinidad, para cuya reducción la Ley Orgánica de Educación ha establecido algunas medidas plasmadas en el Real Decreto 276/2007, el funcionamiento de este sistema de acceso a la docencia no parece cuestionarse en sus aspectos esenciales en nuestro país, más allá de algunas consideraciones puntuales. No obstante, a este respecto es necesario volver a incidir en que son pocos los estudios que han abordado de manera específica esta cuestión. Para profundizar algo más en esta temática, a continuación se presentan dos informaciones de diferente naturaleza. En primer lugar, los resultados de dos encuestas realizadas en el año 2008 en las que se recabaron las opiniones del profesorado y de diversos expertos en educación sobre el acceso a la profesión docente. En segundo lugar, las conclusiones del informe elaborado por la OCDE en el año 2004, en el que se analizaban los procesos de selección del profesorado en el sector público en España.

\subsection{Las opiniones de profesores y expertos sobre los sistemas de acceso a la profesión docente en España}

Durante el año 2008 se dieron a conocer los resultados de dos estudios realizados en España en los que se trataba el tema de los sistemas de acceso a la profesión docente. Ambos trabajos, aunque no se centraron específicamente en esta cuestión, vinieron a cubrir en cierta medida la ausencia de información que, como se ha señalado, existe sobre este tema en nuestro país y nos permiten aproximarnos a las percepciones que el propio profesorado tiene sobre la idoneidad de los procedimientos de ingreso a la enseñanza. En uno de ellos, además, las opiniones del profesorado se complementaron con las de un grupo de expertos, que analizó con mayor detalle los puntos fuertes y débiles del sistema vigente.

El primero de estos estudios (Álvarez Sánchez, 2008), realizado conjuntamente por la Fundación SM y la Organización de Estados Iberoamericanos para la Educación, la Ciencia y la Cultura (OEI), tenía como objetivo general profundizar en la situación de los profesores noveles en España, para lo cual se recabaron las respuestas a un cuestionario cerrado de un to- 
tal de 1.698 profesores de todo el territorio nacional ${ }^{2}$. De acuerdo con las respuestas obtenidas en relación con el actual sistema de oposiciones, el 56\% de la muestra considera que no se trata de un procedimiento adecuado para el ingreso a la docencia, mientras el 63\% está de acuerdo con la idea de que el sistema debería cambiar para tener en cuenta las nuevas funciones que se exigen al profesorado en la actualidad (gráfico 1).

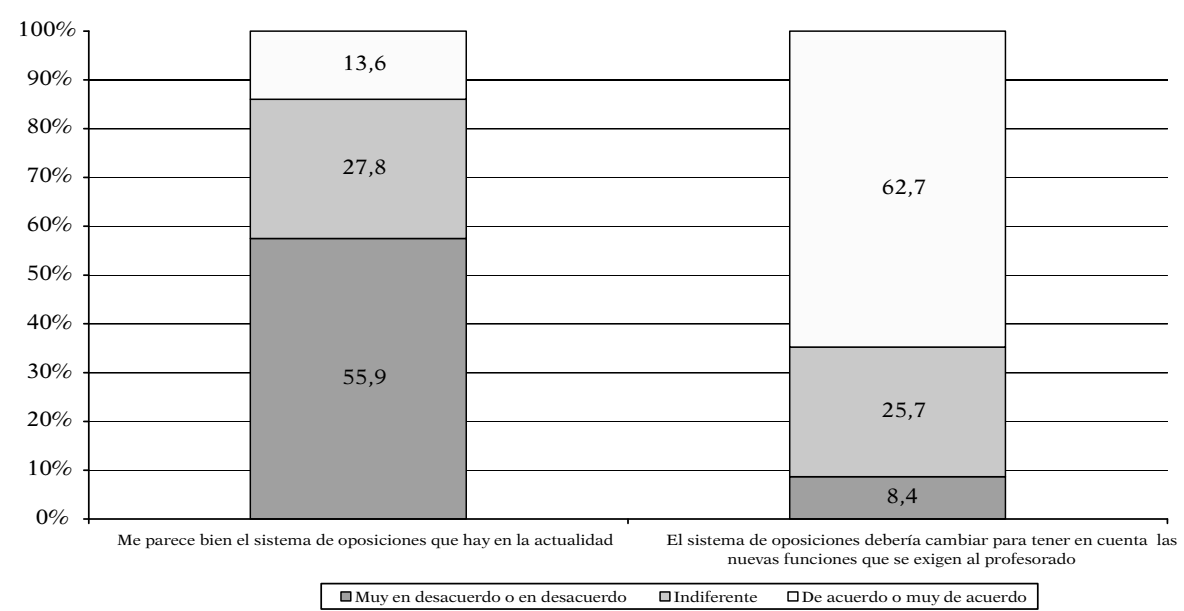

Fuente: Álvarez Sánchez, N., 2008.

Gráfico 1. Opción sobre el sistema de oposiciones.

En relación con el sistema de acceso, el estudio planteaba también la posibilidad de llevar a cabo un curso selectivo teórico-práctico después de superar la oposición o ser contratado por un centro privado. En líneas generales, esta opción no parece convencer al profesorado, ya que tan sólo el $33 \%$ de los docentes se muestra de acuerdo con ella, mientras el $43,5 \%$ se muestra en desacuerdo o muy en desacuerdo. No obstante, cuando se analizan las respuestas en función del número de años de experiencia, el porcentaje que se muestra de acuerdo con este sistema se eleva al $46 \%$ entre los profesores con más de 30 años de antigüedad. También se observan diferencias en función de la etapa educativa, ya que los profesores de secundaria son más favorables al curso selectivo (40\%) que los de Infantil (28\%) y Primaria $(27 \%)$.

Por último, el cuestionario proponía al profesorado la posibilidad de llevar a la práctica un sistema que permitiera a un equipo de profesores acceder a los centros públicos. En este caso el porcentaje de los que consideran positivo ese sistema es del 37,5\%, mientras el 15\% se expresa en contra del mismo. La mayoría de los docentes, sin embargo, se muestran indiferentes ante esta cuestión $(41,5 \%)$. 
El segundo de los estudios que analizó el acceso a la profesión fue una investigación sobre el profesorado en España promovida por la Confederación de Padres de Alumnos (COFAPA) (Pérez Juste; Egido; Sarramona 2008). En este trabajo se elaboró una encuesta a la que respondieron un total de 983 profesores de diferentes niveles educativos y de los distintos sectores de la enseñanza en once Comunidades Autónomas y en la Ciudad Autónoma de Ceuta ${ }^{3}$.

La encuesta citada recoge las opiniones del profesorado sobre la adecuación de los procedimientos que existen en España para ocupar un puesto de trabajo como profesor, diferenciando el sector público y el privado. En términos globales, los resultados muestran que los sistemas vigentes en la actualidad para acceder a la profesión docente son juzgados con tintes negativos por la mayor parte del profesorado, tanto si éstos se refieren a la enseñanza pública como a la privada. El porcentaje de profesores que considera nada o poco adecuado el sistema de ingreso en la enseñanza pública alcanza más de la mitad de las respuestas (el 52\%), mientras el 38\% califica este sistema de adecuado y sólo el $10 \%$ lo considera bastante o muy adecuado. Respecto a los procedimientos de acceso al trabajo en la enseñanza privada, el $57 \%$ considera que son nada o poco adecuados, el 35\% los juzga adecuados y el $8 \%$ bastante o muy adecuados (gráfico 2 ).

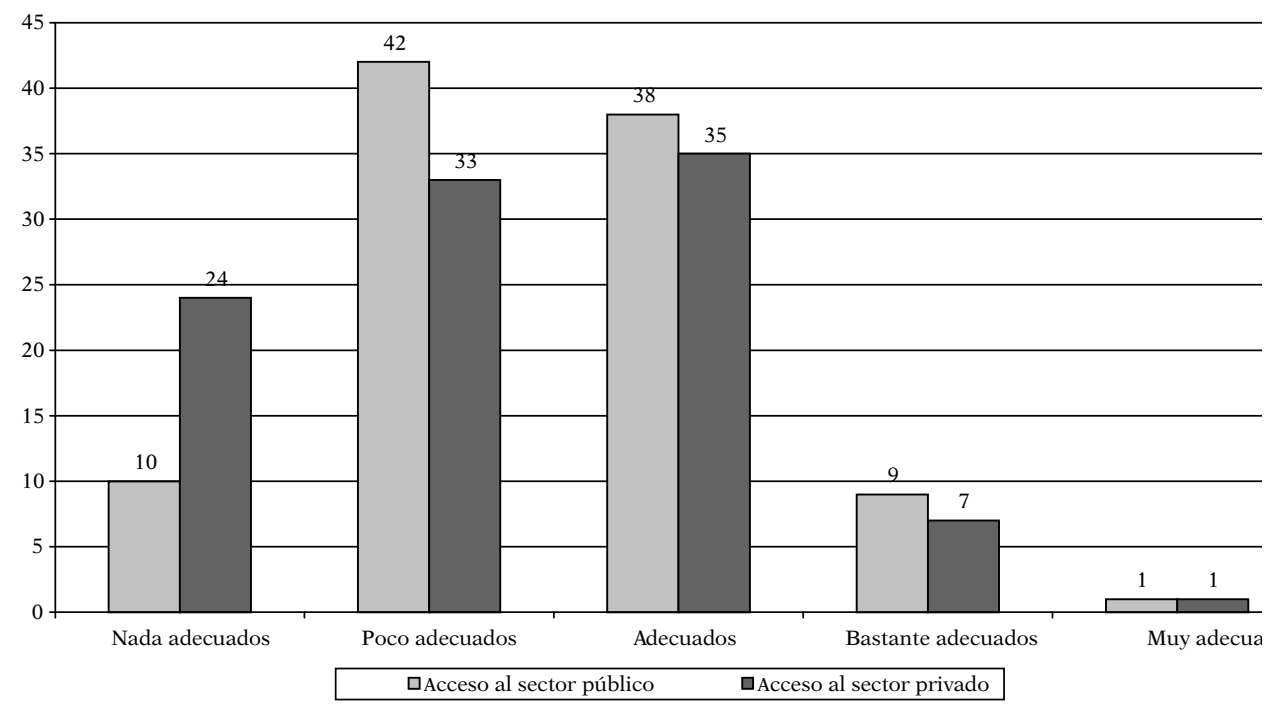

Fuente: (Pérez Juste, R.; Egido, I.; Sarramona, J., 2008).

Gráfico 2. Adecuación de los sistemas de acceso a la docencia.

Opinión del profesorado. 
Como cabe esperar, esta cuestión arroja diferentes resultados si los profesores encuestados pertenecen al sector de la enseñanza pública o al sector de la privada. Así, son los profesores de la enseñanza pública los que valoran más negativamente el sistema de acceso a la profesión en los centros privados (con una media de 1,78 sobre 5), mientras otorgan un "aprobado" al procedimiento de ingreso en la enseñanza pública $(2,55)$. Los profesores de la enseñanza privada también juzgan negativamente el sistema de acceso a la enseñanza pública, con un 2,38, valorando más positivamente el sistema utilizado en su propio sector, con un 2,86 de media (gráfico 3).

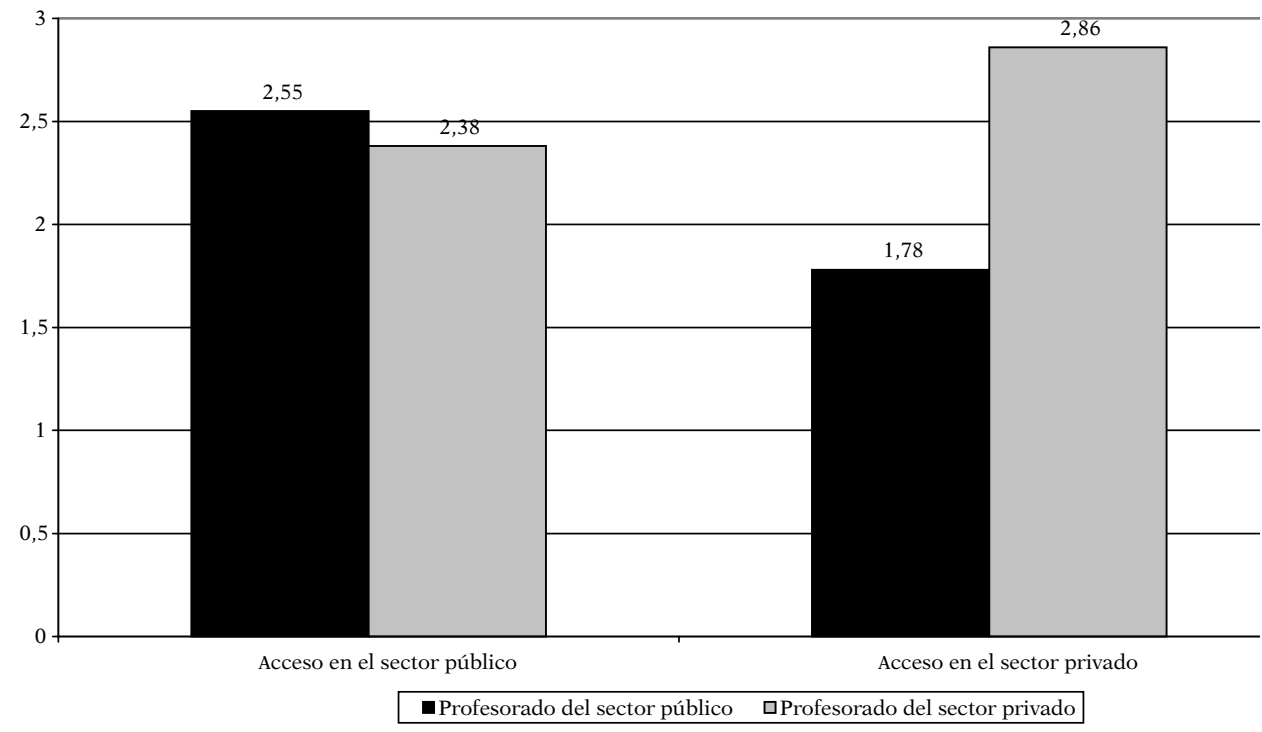

Fuente: (Pérez Juste, R.; Egido, I.; Sarramona, J., 2008).

Gráfico 3. Adecuación de los sistemas de acceso a la docencia. Opinión del profesorado por sector educativo.

Tratando de profundizar un poco más en esta cuestión, la investigación citada recogió también las opiniones de expertos y personas representativas del ámbito de la educación por medio de entrevistas y cuestionarios abiertos. Fueron consultadas más de 30 personas, incluyendo entre ellas a representantes de las administraciones educativas, de los Consejos Escolares Autonómicos y del Estado, de las Asociaciones de padres y madres de alumnos, de las patronales de educación, de sindicatos de profesores, de medios de comunicación y de profesores universitarios especialistas en educación. Las respuestas recogidas en estas entrevistas arrojan también, en términos generales, valoraciones negativas sobre el funcionamiento de los actuales sistemas de acceso a la profesión docente. Así, por lo que se refiere al sector público, la mayoría de los entrevistados enfatiza la rigidez y el carácter 
memorístico de la oposición, señalando que se trata de un sistema inadecuado para seleccionar a personas que realmente tengan aptitudes para la docencia, especialmente en el nivel de secundaria. La oposición se considera, en el mejor de los casos, un procedimiento válido para demostrar conocimientos, pero no un mecanismo que permita comprobar si en la práctica se poseen capacidades para la enseñanza.

A pesar de lo anterior, son también muchos los expertos que señalan en este estudio la dificultad de encontrar alternativas válidas al concurso-oposición, dado que es el mecanismo vigente para la selección de todos los funcionarios públicos y no sólo de los docentes. Además, se considera que con él se garantizan, al menos, unas ciertas condiciones de igualdad. Por estas razones, varias de las personas entrevistadas proponen la reformulación del actual sistema de oposiciones, más que el abandono del mismo. En esos casos, se subraya la necesidad de que los exámenes otorguen un mayor peso a la práctica docente. De igual modo, se considera pertinente la puesta en funcionamiento de procesos de evaluación del desempeño profesional de los candidatos seleccionados, de los que dependa su posterior nombramiento definitivo como profesores.

Ahondando algo más en esta cuestión, varios de los expertos critican el acceso a la profesión de los docentes de la enseñanza pública por medio de contratos de interinidad. En este sentido, recalcan el hecho de que muchos profesores están ejerciendo sin haber superado previamente la oposición. Algunos entrevistados señalan, además, que la existencia de listas de interinos más o menos amplias se deja al arbitrio de las administraciones educativas, que aumentan o reducen el número de profesores que se encuentran en ellas en función de su propia conveniencia política y económica en un momento concreto.

Por lo que se refiere al sistema de acceso a la enseñanza privada, las opiniones de los expertos entrevistados muestran una mayor división. Para algunos, es razonable que los centros privados actúen en este ámbito con sus propios criterios, seleccionando a los profesores que crean oportuno, como lo hace cualquier empresa privada. Sin embargo, son también muchos los entrevistados que señalan la excesiva arbitrariedad que subyace en este sistema, en el que los criterios de selección pueden encontrarse alejados de los principios de mérito y capacidad.

Además de lo anterior, puede señalarse la diferenciación que varias de las personas que respondieron a las entrevistas y cuestionarios establecen entre la enseñanza privada y la enseñanza concertada en relación con esta cuestión. En el sector de la enseñanza privada no subvencionada el principio de libertad de empresa a la hora de seleccionar al personal docente no se 
cuestiona, pero la situación es distinta cuando se habla de la enseñanza concertada. En este caso, al tratarse de puestos de trabajo financiados con dinero público, varios representantes de la administración y de los sindicatos opinan que el sistema de acceso debería homogeneizarse con el de la enseñanza pública. De hecho, para algunos de ellos los mecanismos de acceso a la profesión en estos centros deberían vincularse a la equiparación del salario y de las condiciones laborales de los profesores de este sector con los del sector público.

\subsection{Las recomendaciones de la OCDE sobre el acceso a la profesión docente en España}

En el marco del proyecto denominado Atraer, formar y retener profesores eficaces, la OCDE realizó en el año 2004 un informe sobre el profesorado en España, en el que se trató de manera específica el acceso a la docencia en la enseñanza pública (Cros; Duthilleul; Cox; Kantasalmi, 2004). En líneas generales, este informe consideró que el sistema de concurso-oposición vigente en nuestro país podía calificarse de adecuado. No obstante, puso también de manifiesto algunas debilidades del proceso de selección y señaló posibles líneas de mejora del mismo.

En concreto, las críticas planteadas en este trabajo hacían referencia al hecho de que el concurso-oposición, tal y como está diseñado en España, otorga un peso mucho mayor a los conocimientos académicos de los futuros profesores que a sus capacidades o habilidades para la enseñanza, por lo que no se cumple el objetivo de seleccionar a las personas más cualificadas para el trabajo docente. El estudio señalaba también como un aspecto negativo del sistema español el hecho de que la fase de prácticas, que en teoría forma parte del proceso de selección, tiene en realidad un carácter puramente formal, puesto que todos los candidatos la superan de forma casi automática. Además, en opinión de los expertos de la OCDE, las oposiciones no sirven para mejorar la formación inicial del profesorado en España, puesto que no se basan en un perfil estandarizado de carácter nacional que delimite lo que los profesores deben ser capaces de hacer.

A partir de esas críticas, la OCDE formuló algunas recomendaciones para mejorar la selección de los docentes en España. En concreto, las propuestas que reflejó el informe fueron las siguientes:

- Resulta necesario realizar una modificación en los temarios sobre los que versan los exámenes de las oposiciones, centrándolos fundamentalmente en las habilidades y competencias que los profesores necesitan para resolver problemas pedagógicos concretos. 
- Sería conveniente reducir el número de temas que se incluyen en los exámenes, con el fin de ganar profundidad en el tratamiento de los mismos.

- El sistema debería contar con criterios claros y explícitos para la corrección de los exámenes, traducidos a indicadores, de forma que puedan evaluarse en condiciones de igualdad los resultados de todos ellos, reduciendo la posible subjetividad o diferencia de criterios entre los distintos tribunales.

- Un requisito esencial es la reforma del periodo de prácticas, que debe constituir un elemento esencial de la selección, asumiendo que no todos los aspirantes están preparados para superarlo.

Complementando estas recomendaciones, las sugerencias del informe de la OCDE para las políticas de acceso del profesorado en España se centraron en la necesidad de desarrollar perfiles de selección específicos para puestos docentes concretos. Dichos perfiles, basados en las características de los centros escolares, deberían servir de base para la adjudicación de las plazas vacantes, en lugar de utilizar como único criterio la puntuación obtenida en el concurso-oposición. Puesto que las escuelas tienen necesidades distintas y proyectos diferentes, este sistema permitiría que el profesorado se ajustara mucho mejor a las peculiaridades de cada centro.

\section{ALGUNAS REFLEXIONES SOBRE EL SISTEMA DE ACCESO A LA PROFESIÓN DOCENTE EN ESPAÑA}

A partir de las informaciones aportadas en el punto anterior, y teniendo en cuenta la visión general del panorama europeo, conviene detenerse a reflexionar, aunque sea brevemente, sobre la posibilidad de mejorar el sistema de acceso a la profesión docente en España. Como se ha mencionado al comienzo del artículo, se trata de un momento oportuno para plantear ese objetivo, puesto que los reglamentos de acceso a los cuerpos docentes deberán ser modificados una vez que finalice el periodo de implantación de la Ley Orgánica de Educación y empiecen a egresar de las universidades los titulados que hayan cursado el nuevo sistema de formación inicial del profesorado.

Además de lo anterior, también conviene tener presente que el Estatuto del Funcionario Docente no universitario aún se encuentra pendiente de aprobación. Dicho Estatuto pretende contemplar la carrera docente en su conjunto, por lo que debe incluir los procedimientos de selección del profesorado. El último Borrador del Estatuto, que fue presentado en mayo del 
año 2007 por el Ministerio de Educación (MEC, 2007), planteaba mantener el sistema de acceso a la enseñanza pública mediante concurso-oposición. No obstante, la formulación del texto del Borrador en relación con este tema era lo suficientemente amplia como para permitir la introducción de modificaciones respecto del sistema actual.

En esta situación, y con las pautas que pueden deducirse de lo tratado en los epígrafes anteriores, cabe plantear a modo de conclusiones algunas posibles líneas de mejora de los procedimientos de acceso a la docencia en nuestro país. El objetivo no es definir en este momento un conjunto de propuestas cerradas, sino más bien proponer algunas cuestiones que deberían debatirse en profundidad para perfeccionar los procedimientos de selección del profesorado.

Una primera cuestión que convendría tratar a este respecto se deriva del sistema que se utiliza en un buen número de los países europeos, en los que se establecen procesos de selección del profesorado previos al acceso a la formación inicial. La existencia de algún mecanismo que permitiera garantizar que en las Facultades de Educación se matriculan los estudiantes adecuados, no sólo en términos de rendimiento, sino, sobre todo, en términos de su capacidad para llevar a cabo el trabajo con los alumnos, sería, sin duda, un factor muy positivo para contar con un profesorado de calidad. No es sencillo, en el caso de España, hablar de procedimientos selectivos para el acceso a la formación docente, ya que se trata de un tema controvertido, que afecta a los sistemas de ingreso en la enseñanza universitaria (Egido, 2008). Sin embargo, la implantación de medidas similares a las que se emplean en el entorno europeo solventaría considerablemente muchos de los problemas que se derivan de la actual masificación de la formación inicial del profesorado y simplificaría el acceso posterior al ejercicio profesional.

La segunda reflexión, relativa al sistema de ingreso en el sector público, parte de la base de que el concurso-oposición puede considerarse adecuado en términos generales. Sin embargo, en relación con este sistema existen algunos aspectos que deberían replantearse. Tanto los resultados de los estudios empíricos realizados por la Fundación SM y la OEI y por la Confederación de Padres de Alumnos como las recomendaciones formuladas por los especialistas de la OCDE son claros a este respecto: las oposiciones deberían dar mucho más peso a las capacidades docentes y a las habilidades didácticas de los aspirantes y centrarse más en las exigencias que la sociedad actual plantea al profesorado. Se trata, por tanto, de primar en las pruebas la posesión de las competencias profesionales de carácter docente, en lugar de atender a la reproducción de los conocimientos relacionados con las disciplinas académicas adquiridos durante la formación. De esta manera, además, los procedimientos de acceso serían más coherentes con el modelo de 
formación inicial del profesorado recientemente implantado, que se basa precisamente en un conjunto de competencias que los profesores de cada una de las etapas deben alcanzar ${ }^{4}$.

Además de lo anterior, la fase de prácticas tras el concurso-oposición debería ser objeto de una atención especial, considerando que se trata de una etapa fundamental en el proceso selectivo. Con este fin, sería necesario reformular toda la organización del periodo de prácticas de los funcionarios, estableciendo criterios precisos de control de su funcionamiento y sistemas eficaces de evaluación de los futuros docentes, así como incentivos para los profesores que actúan como tutores.

Junto a los cambios en los procedimientos selectivos, sería conveniente introducir también algunas modificaciones en el sistema de adjudicación de las plazas vacantes. En este aspecto, se trataría de conjugar que sean los mejores candidatos los que superen las oposiciones con el requisito de que el acceso a un puesto docente concreto tenga en cuenta las necesidades y los proyectos educativos de los centros. Probablemente, la elaboración de perfiles de acceso, en la línea de lo sugerido por la OCDE, podría contribuir a la mejora del sistema actual. Dichos perfiles, formulados por los centros en función de las características específicas de sus plazas vacantes, permitirían tener en cuenta, además de las puntuaciones de los aspirantes en el concurso-oposición, algunos factores relacionados con su formación, su experiencia previa o sus motivaciones profesionales.

Por otra parte, no hay que olvidar que los funcionarios docentes representan sólo una parte del profesorado de la enseñanza pública en nuestro país, ya que la proporción de docentes interinos ha llegado a alcanzar cifras muy altas en algunos momentos. Es necesario realizar cambios en el sistema selectivo para evitar que se produzca este desajuste en la oferta de empleo para el profesorado. Es cierto que en los últimos años se han dado pasos para solucionar este problema, pero es importante establecer mecanismos para que en el futuro esta situación no vuelva a repetirse.

Además de lo anterior, y aunque en este terreno los datos son más escasos y muestran una mayor división, conviene reflexionar también sobre los sistemas de acceso a la docencia en el sector privado. En términos generales, los sistemas de ingreso a la enseñanza privada no parecen ponerse en cuestión, aunque existen algunas voces discrepantes en el caso de los centros concertados. Parece lógico que en esos centros se garantice la afinidad del profesorado con el ideario escolar, pero también es importante salvaguardar los principios de mérito, capacidad e igualdad en el acceso a la profesión docente, dado que se trata de puestos de trabajo financiados con recursos públicos. La legislación ya preveía para tal fin la existencia de las comisiones de 
selección, pero es posible que en este aspecto las garantías de transparencia en la contratación deban ampliarse.

En cualquier caso, es evidente que los procedimientos de acceso a la docencia no pueden contemplarse de una forma aislada, sino que deben considerarse en estrecha relación con el sistema de formación inicial y con el conjunto de la carrera profesional de los profesores. Por ese motivo, las reflexiones apuntadas más arriba deberían inscribirse en un debate más amplio, que incluyera de una forma global todo el conjunto de factores que configuran la profesión docente en nuestro país. 


\section{NOTAS}

1. Los sindicatos se movilizan contra el MEC. Comunidad Escolar, 795, 11 de octubre de 2006.

2. El 12\% de los cuestionarios corresponde a profesores de Educación Infantil, el 34\% a Primaria y el $44 \%$ a Secundaria. El $62 \%$ son profesores de la enseñanza pública y el $38 \%$ de la privada-concertada. El 33\% de los docentes que cumplimentaron el cuestionario tenía menos de 11 años de experiencia profesional, el $23 \%$ entre 11 y 20 años y el $43 \%$ más de 20 años.

3. La muestra estaba formada por un $43,5 \%$ de varones y un $56,5 \%$ de mujeres. Por niveles educativos, el 13\% ejercía en Educación Infantil, el 25\% en Primaria y el $62 \%$ restante en Secundaria (ESO, Bachillerato y FP). El 55\% de los encuestados corresponde a centros públicos, el $40 \%$ a centros concertados y el $5 \%$ a centros privados. Las Comunidades Autónomas incluidas en el estudio fueron Andalucía, Aragón, Cantabria, Castilla-La Mancha, CastillaLeón, Extremadura, Galicia, La Rioja, Madrid, Murcia y Navarra.

4. Dichas competencias han sido definidas en las Órdenes Ministeriales que establecen los requisitos para la verificación de los títulos universitarios oficiales que habilitan para el ejercicio de la profesión docente. Para Infantil la ORDEN ECI/3854/2007, para Primaria la ORDEN ECI/3857/2007 y para Secundaria la ORDEN ECI/3858/2007. 


\section{REFERENCIAS BIBLIOGRÁFICAS}

Álvarez Sánchez, N. (2008). La situación de los profesores noveles. Madrid: OEIFundación SM.

Barber, M.; Mourshed, M. (2007). How the World Best-performing School Systems Come out on Top. McKinsey \& Company [en línea]. Disponible en: http://www.mckinsey.com/locations /ukirland/publications/pdf/Education_ report.pdf [consulta 2008, 30 de julio].

Cros, F.; Duthilleul, Y.; Cox, C.; Kantasalmi, K. (2004). Attracting, Developing and Retaining Effective Teachers. Country Note: Spain [en línea] Paris: OECD. Disponible en: http://www. oecd.org/dataoecd/37/41/32185669.pdf [consulta 2008, 15 de junio].

Darling-Hammond, L. (2000). Teacher Quality and Student Achievement: A Review of State Policy Evidence. Educational Policy Analysis Archives [en línea] 8, (1). Disponible en: http://epaa. asu.edu/epaa/v8n1 [consulta 2008, 12 de julio].

Dávila, P. (1988). Las oposiciones al magisterio y la normalización de los saberes pedagógicos. Revista de Educación, 286, 115-127.

Egido, I. (2008). La formación inicial del profesorado: evolución histórica, prospectiva y controversias, en Valle, J. M. (dir.) El pacto escolar: la necesidad de un consenso nacional en materia de educación. Bilbao: Fundación para la Libertad.

Esteve, J. M. (1984). Profesores en conflicto. Repercusiones de la práctica profesional sobre la personalidad de los enseñantes. Madrid: Narcea.

Eurydice (2002). La profesión docente en Europa: perfil, tendencias e intereses.
Temas clave de la educación en Europa, volumen 3. Informe II: Oferta y demanda. Educación Secundaria Inferior General. Bruselas: Unidad Europea de Eurydice.

Eurydice (2004). La profesión docente en Europa: perfil, tendencias e intereses. Temas clave de la educación en Europa. Volumen 3. Informe IV: el atractivo de la profesión docente en el siglo XXI. Bruselas: Unidad Europea de Eurydice.

Eurydice (2005). La profesión docente en Europa: perfil, tendencias e intereses. Temas clave de la educación en Europa. Volumen 3. Informe Anexo: reformas de la profesión docente: análisis histórico (1975-2002). Bruselas: Unidad Europea de Eurydice.

Eurydice (2008). The Education System in France: 2007/2008 [en línea]. Brussels: Eurydice. Disponible en: http:// eacea.ec.europa.eu/ressources/eurydice /eurybase/pdf/0_integral/FR_EN.pdf [consulta 2008, 2 de junio].

Fernández Tilve, M. D.; Malvar, L. (2000). Una aproximación a los procesos selectivos de la profesión docente: el caso de la enseñanza pública en el contexto gallego. Enseñanza, 17-18, 281-305.

Gabriel, N. de (1994). La formación del magisterio, en Guereña, J. L.; Ruiz Berrio, J; Tiana, A. (eds.) Historia de la Educación en la España contemporánea. Diez años de investigación. Madrid: CIDE, 215-265.

Ley Orgánica 8/1985 Reguladora del Derecho a la Educación (BOE 19 de octubre de 1985).

Ley Orgánica 2/2006, de 3 de mayo, de Educación (BOE 4 de mayo de 2006). 
Ministerio de Educación y Ciencia (2007). Borrador del Estatuto del Funcionario Docente no universitario [en línea]. Madrid: MEC. Disponible en http://www.periodicoescuela.es/infoesc uela/pdf/BorradorEstatutoMayo07.pdf [consulta 2008, 14 de junio].

Montero, M. L. (2006). Profesores y profesoras en un mundo cambiante: el papel clave de la formación inicial. $R e$ vista de Educación, 340, 66-86.

Murillo, J. (coord.) (2007). Carrera y evaluación del desempeño docente. Un estudio comparado entre 50 países de América y Europa. Santiago de Chile: Oficina Regional de Educación para América Latina y el Caribe/UNESCO, $2^{\mathrm{a}}$ ed.

OECD (2004). Teachers Matter: Attracting, Developing and Retaining Effective Teachers. Paris: OECD.

ORDEN ECI/3854/2007, de 27 de diciembre, por la que se establecen los requisitos para la verificación de los títulos universitarios oficiales que habiliten para el ejercicio de la profesión de Maestro en Educación Infantil (BOE 29 de diciembre de 2007).

ORDEN ECI/3857/2007, de 27 de diciembre, por la que se establecen los requisitos para la verificación de los títulos universitarios oficiales que habiliten para el ejercicio de la profesión de Maestro en Educación Primaria (BOE 29 de diciembre de 2007).

ORDEN ECI/3858/2007, de 27 de diciembre, por la que se establecen los requisitos para la verificación de los títulos universitarios oficiales que habiliten para el ejercicio de la profesión de Profesor de Educación Secundaria Obligatoria y Bachillerato, Formación Profesional y Enseñanzas de Idiomas (BOE 29 de diciembre de 2007).

Peretti, A. (1982). La formation des personnels de l'éducation nationale: rapport au ministre de l'Éducation nationale de la Commission de la formation des personnels de l'éducation nationale. Paris: La Documentation française.

Pérez Juste, R. ; Egido, I ; Sarramona, J. (2008). Respaldo social a la profesión docente [en línea]. Madrid: Confederación de Padres de Alumnos (COFAPA). Disponible en: http://www.cofapa. net/docs/1226665575_Ponencia\%20I.E gido.pdf [consulta 2008, 14 de noviembre].

Real Decreto 276/2007, de 23 de febrero, por el que se aprueba el Reglamento de ingreso, accesos y adquisición de nuevas especialidades en los cuerpos docentes a que se refiere la Ley Orgánica 2/2006, de 3 de mayo, de Educación, y se regula el régimen transitorio de ingreso a que se refiere la disposición transitoria decimoséptima de la citada ley (BOE 2 de marzo de 2007).

Requena, M. M. (2007). Reflexiones en torno al sistema de oposiciones a la docencia primaria en España y las funciones éticas de los docentes. Cuestiones Pedagógicas, 18, 301-324.

Wall, W. D. (1959). Education et Santé Mentale. Paris: UNESCO. 


\section{PALABRAS CLAVE}

Selección de profesores. Acceso al empleo. Profesión docente. Unión Europea.

\section{KEY WORDS}

Teachers recruitment. Employment access. Teaching profession. European Union.

\section{PERFIL ACADÉMICO DE LA AUTORA}

Inmaculada Egido Gálvez es Doctora en Ciencias de la Educación y Profesora Titular de Universidad en el Departamento de Teoría e Historia de la Educación de la Universidad Complutense de Madrid. Su actividad investigadora se centra en el área de la política educativa comparada. Es autora de varios libros y artículos sobre la formación, selección y condiciones laborales del profesorado, temas en los que también ha colaborado con diversos organismos internacionales.

Dirección de la autora: Dpto. de Teoría e Historia de la Educación.

Facultad de Educación

Universidad Complutense de Madrid

C/ Rector Royo Villanova s/n

28040 Madrid

E-mail: miegido@edu.ucm.es

Fecha de Recepción del artículo: 13. Enero. 2009

Fecha de Revisión del artículo: 04. Febrero. 2010

Fecha de Aceptación del artículo: 10. Febrero. 2010 